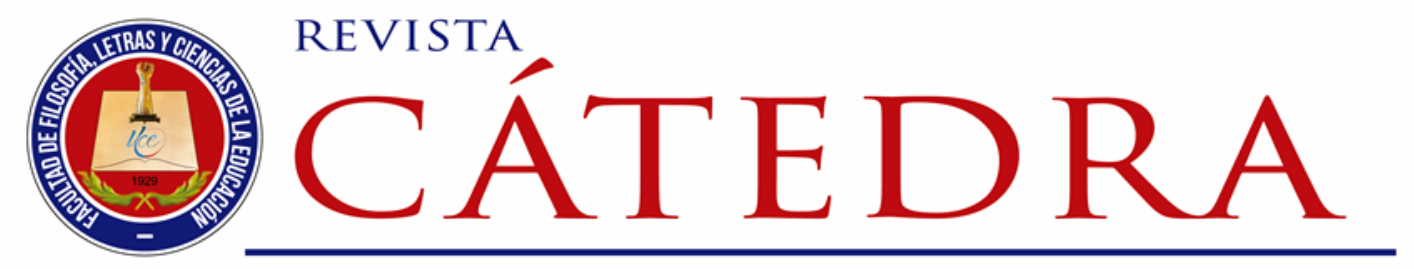

\title{
Incidencia de los factores personales, ambientales, y de interacción social en la deserción escolar en educación a distancia - virtual
}

\section{Incidence of personal, environmental, and social interaction factors in school dropout in distance - virtual education}

\author{
Ángel Vivanco-Saraguro \\ Universidad Andina Simón Bolívar, Quito, Ecuador \\ angel.vivanco@uasb.edu.ec \\ https://orcid.org/0000-0001-5496-7733
}

(Recibido: 12/06/2020; Aceptado: 18/06/2020; Versión final recibida: 07/08/2020)

Cita del artículo: Vivanco-Saraguro, A. (2020). Incidencia de los factores personales, ambientales, y de interacción social en la deserción escolar en educación a distancia virtual. Revista Cátedra, 3(3), 111-128.

\section{Resumen}

La deserción escolar es, probablemente, el mayor problema que afecta a los sistemas de educación a distancia - virtual. Por ello, estudios contemporáneos mencionan que es necesario investigar con sentido crítico los factores que generan esta problemática. En este sentido, en el programa del Bachillerato a Distancia - Virtual de la Unidad Educativa Juan Montalvo de la ciudad de Quito, como unidad comprometida con la educación de personas jóvenes y adultas con escolaridad inconclusa, se planteó como objetivo evaluar la incidencia de los factores personales, ambientales, y de interacción social en la deserción escolar de este programa. El estudio se desarrolló bajo un enfoque cuantitativo de corte correlacional, y para el levantamiento de la información se aplicó un cuestionario en línea a estudiantes de primer y tercer año de Bachillerato General Unificado. El análisis de los resultados se realizó con el 5\% de error, y se aplicó la prueba Chi-Cuadrado para determinar la independencia o correlación entre las variables. Los resultados indicaron que la mayoría de los factores personales y ambientales no inciden de manera significativa en la deserción

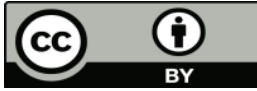


escolar. Mientras que, los factores de interacción social, en su mayoría, demostraron incidir significativamente en la decisión de desertar o abandonar los estudios.

\section{Palabras clave}

Deserción escolar, educación a distancia, educación virtual, factores personalesambientales, interacción social,

\section{Abstract}

Dropout is probably the biggest problem affecting distance - virtual education systems. For that reason, contemporary studies mention that it is necessary to investigate with a critical sense the factors that generate this problem. In this sense, in the program of the Distance Virtual Baccalaureate of the Juan Montalvo Educational Unit of the city of Quito, as a unit committed to the education of young people and adults with unfinished schooling, the objective was to evaluate the incidence of personal, environmental, and social interaction factors in the school dropout of this program. The study was developed under a quantitative approach of correlation, and an online questionnaire was applied to first and third year students of the Unified General Baccalaureate. The analysis of the results was made with a $5 \%$ error, and the Chi-square test was applied to determine the independence or correlation between the variables. The results indicated that most of the personal and environmental factors do not have a significant influence on school desertion. While, most of the social interaction factors showed to have a significant influence on the decision to drop out or to abandon the studies.

\section{Keywords}

School dropout, abandonment, personal and environmental factors, social interaction, distance education, virtual education

\section{Introducción}

Un estudio contemporáneo en el contexto educativo ecuatoriano menciona que la deserción escolar es una latente problemática que atenta contra el sistema educativo, el estudiantado, y el desarrollo del país (Posligua, 2019). De hecho, la deserción escolar en cualquier nivel y modalidad educativa representa un costo social y económico muy alto, tanto para los estudiantes, como para las instituciones educativas, y finalmente, para la sociedad en general (Morfin, González y Palomera, 2018).

Esta problemática se agrava aún más en los programas de educación a distancia ya que, si bien matriculan más estudiantes, afrontan también mayores índices de deserción que la modalidad presencial. Al respecto, Acuña (2018), menciona que los niveles de deserción escolar, suelen ser un $20 \%$ mayor en los programas educativos a distancia que en la educación convencional.

Por décadas, investigadores han estudiado los factores detonantes de la deserción en la educación a distancia, en su mayoría a nivel de educación superior. En la Universidad Nacional Abierta y a Distancia de Colombia, la falta de recursos económicos y la incompatibilidad horaria son los factores de mayor peso en la deserción que fluctúa entre el 40 y 60\% (Peralta y Mora, 2016). Un programa del Instituto de Aprendizaje en Línea, en México, reportó que los factores académicos, principalmente, el bajo rendimiento escolar, generó una deserción del 61\% (Morfin, González y Palomera, 2018). De igual forma, el programa en línea de Nivelación a la licenciatura en Trabajo Social de la Universidad de 
Guadalajara acusó un 40\% de deserción estudiantil, relacionada primordialmente a factores personales, como enfermedades, situación laboral, entre otros (De Aguinaga y Barragán, 2007).

Aun cuando el problema de la deserción escolar se viene estudiando desde hace varios años, y en diferentes modalidades educativas, es necesario mencionar que no existen muchos estudios o investigaciones que proporcionen suficiente información al respecto (Peña y Rodríguez, 2019). Pocos son los trabajos enmarcados a la deserción en el nivel de bachillerato en modalidad a distancia, y más escasos aún son los estudios que se centren en la deserción escolar en bachilleratos en modalidad a distancia - virtual en Ecuador.

Al respecto, en Ecuador, el Ministerio de Educación oferta el Bachillerato a Distancia Virtual a través de la Unidad Educativa Juan Montalvo de la ciudad de Quito. Este programa se desarrolla mediante la plataforma educativa Moodle, de forma totalmente virtual y atiende a personas jóvenes y adultas con rezago escolar, brindándoles la oportunidad de culminar sus estudios secundarios. No obstante, pese a que los índices de matrícula incrementan cada período, existe una tasa de deserción escolar que preocupa a docentes, coordinadores y autoridades. Cabe mencionar que actualmente no existen todavía datos oficiales de dominio público concernientes a los niveles de deserción escolar de este programa, como tampoco un estudio que averigüe las causas que generan este problema.

Todos los antecedentes hasta aquí mencionados motivaron la realización de esta investigación, pues permitió analizar la deserción escolar del Bachillerato a Distancia Virtual, y su relación con los factores personales, ambientales y de interacción social. Además en la Unidad Educativa Juan Montalvo de la ciudad de Quito, no se ha desarrollado ningún estudio que investigue las causas que generan este problema. Sin duda, identificar los factores por los cuáles desertan los estudiantes del Bachillerato a Distancia - Virtual es un buen comienzo para comprender esta problemática.

Con lo expuesto hasta este punto, el objetivo del presente trabajo es evaluar la incidencia de los factores personales, ambientales, y de interacción social en la deserción escolar del programa de Bachillerato a Distancia - Virtual. Para ello, en la primera parte del artículo se presenta un breve acercamiento teórico al concepto de deserción escolar, a los modelos que permiten comprender el fenómeno, y se explica el marco teórico utilizado en este trabajo. En la segunda parte, se detalla la metodología empleada para la obtención y análisis de los datos. En la tercera parte, se exponen los principales resultados. Luego, en la cuarta parte, se discuten los resultados del estudio, para finalmente, en la quinta parte, señalar las conclusiones más relevantes del trabajo.

\subsection{Deserción escolar}

El término deserción, según la Real Academia Española, proviene del latín desertio que significa abandono. En este sentido, se puede calificar de desertor a aquella persona que renuncia, desiste o abandona una actividad, meta u objetivo que lo mantenía vinculado a una institución (González, 2002). Dicho de otra manera, deserción es un concepto que indica cese o retiro de un proceso.

En el contexto educativo, la deserción escolar se presenta cuando el estudiante abandona todo tipo de relación que de alguna manera lo mantenía ligado a la institución educativa (González, 2002). Este abandono puede ser considerado un fenómeno multifactorial, ya que es provocado por una combinación de factores que se originan tanto en la institución educativa, como en los entornos de tipo individual, familiar y social (Venegas et ál., 2017). 
Al respecto, Muñoz (2013), menciona que la deserción escolar es un acto donde el estudiante se desvincula del sistema educativo producto de causas de diferente naturaleza. Esta aseveración converge con la de Tinto (1982), quien define a la deserción escolar como una situación que enfrenta el estudiante cuando abandona el proceso escolar por una o varias razones, y no logra concluir su proyecto educativo.

Por su parte, en Ecuador, el Ministerio de Educación define a la deserción como el número de estudiantes que abandonaron un curso antes de su culminación, con respecto al total de matriculados en ese año escolar (Antamba, 2015). Esta última definición deja ver que, en ocasiones, el término deserción se asocia, únicamente, con el indicador que mide el ausentismo o abandono de los estudiantes a las instituciones educativas (Muñoz, 2013).

Con lo anterior, precisar de forma puntual una definición para deserción escolar resulta un tanto dificultoso (Muñoz, 2013), debido a que varios autores la han definido desde diferentes enfoques. Sin embargo, existe consenso para definirla como el abandono o ausentismo del estudiante al sistema educativo, dejando de lado toda formación académica (Pugo, 2017). En todo caso, ninguna definición expresa en su totalidad la complejidad de este problema, por ello cada investigador adopta la definición que mejor se acople a sus objetivos y al problema a investigar (Tinto, 1989).

\subsection{Modelos teóricos que explican la deserción escolar}

Para el estudio de la deserción escolar en general existen diferentes modelos teóricos que intentan explicar las razones de por qué los estudiantes abandonan o desertan de una institución educativa. La mayor parte de las denominadas teorías se presentan organizadas a manera de modelos, en los cuales relacionan diversas variables y acontecimientos que explican y describen determinados comportamientos de los estudiantes (Cruz, 2003).

Uno de los autores más citados en estudios referentes al tema es Vincent Tinto, quien, basado en el trabajo de Durkheim, menciona que las condiciones que llevan a una persona al suicidio son similares a las que conducen al estudiante a la deserción escolar (Benítez, 2016). Tinto (1989), luego de un exhaustivo análisis de estudios concernientes al tema, clasifica los factores que precipitan la deserción en cinco marcos: 1) psicológicos; 2) sociales; 3) económicos; 4) organizacionales; e, 5) interaccionales.

El primero aborda variables psicológicas como la personalidad, la motivación, y habilidades propias de cada estudiante. Al segundo marco conciernen factores como el estatus social y el género, como predictores del ascenso social y el éxito escolar. En el marco económico se establecen las causas que los estudiantes manifiestan en cuanto a los costos de sus decisiones en relación a sus ingresos, este componente es crucial a la hora de decidir la permanencia escolar. Por lo que respecta a los descriptores del marco organizacional, refieren las variables atribuibles a las instituciones educativas, tales como la administración, la infraestructura, los recursos con los que cuenta, los docentes, etc. Por último, en el marco interaccional se sitúa la reciprocidad que puede darse entre el ámbito personal y el organizacional.

La visión de Vincent Tinto es una perspectiva general que ha servido como punto de partida para el resto de modelos teóricos que se han desarrollado por autores dedicados al tema de la deserción escolar. Dichos modelos presentan variaciones en su organización, pero, conceptualmente, mantienen la esencia de esta visión general con leves modificaciones en función del contexto escolar bajo estudio.

Licencia Creative Commons Atribución 4.0 Internacional (CC BY 4.0)

Revista Cátedra, 3(3), pp. 111-128, septiembre-diciembre 2020. e-ISSN:2631-2875

https://doi.org/10.29166/catedra.v3i3.2279 
Por ejemplo, Espíndola y León (2002), establecen dos marcos para interpretar los factores que precipitan la deserción escolar, un marco extrasistema y un marco intrasistema. El primero aborda factores de la situación socioeconómica y del contexto familiar de los estudiantes, en tanto que, el segundo, contempla factores institucionales, académicos y docentes. Así también, autores como Vásquez y Rodríguez (2007), concuerdan y sintetizan los factores que influyen en la deserción escolar en cuatro grupos, individuales, socioeconómicos, institucionales y académicos.

Igualmente, en el campo de la educación a distancia, García (2019), propone tres marcos para explicar los factores que influyen en la deserción escolar: 1) factores atribuibles al estudiante; 2) factores atribuibles a los docentes; $y, 3$ ) factores atribuibles a la institución educativa. En ese mismo sentido, Frankola (2001), de manera más detallada, menciona que factores como la ausencia de acompañamiento, poca motivación, dificultades con la tecnología, falta de apoyo, diseño inadecuado de los cursos y tutores inexpertos, son las principales causas que inciden en la deserción escolar en la educación a distancia virtual.

En este punto conviene señalar que, actualmente, existen diversos modelos desarrollados por autores que permiten explicar el fenómeno de la deserción; no obstante, hay un grado de consenso para establecer las variables relacionadas con esta problemática.

\subsection{Modelo teórico utilizado en este trabajo}

Para esta investigación se tomó como referente teórico el modelo de Rivera (2011), quien, basándose en teorías, investigaciones y modelos anteriores, sugiere un modelo que agrupa en cinco categorías los factores que promueven la decisión de desertar o permanecer en los estudios a distancia, estas son:

- Personales. Entre estos se encuentra el sexo, la edad, el estado civil, el número de hijos, la zona de residencia, la situación laboral, el nivel de ingresos, la aspiración educativa, entre otros.

- Académicos. Tales como recibir consejería académica, la calidad del apoyo académico, los hábitos de estudio, la pertinencia y calidad de los contenidos de aprendizaje, el rendimiento escolar, el proceso de evaluación, entre otros.

- Ambientales. En esta categoría se consideran los factores relacionados a los recursos necesarios para financiar los estudios, la carga laboral del estudiante, el apoyo que recibe de otras personas, las responsabilidades familiares y su relación con los estudios, trabajo y familia, entre otros.

- Interacción social. Entre estos se encuentra la calidad de las interacciones en el curso con los docentes y entre compañeros, la retroalimentación de los docentes en relación al progreso académico, la disponibilidad de los profesores mediante las vías de comunicación que provee la plataforma educativa, la motivación que transmiten los docentes y la institución; entre otros.

- Apoyo tecnológico. Esta categoría considera factores como el manejo de la plataforma, la efectividad de los recursos que esta dispone, la disponibilidad y pertinencia de los materiales, el apoyo y guía que reciben los estudiantes al iniciar el curso, las habilidades tecnológicas de los estudiantes, entre otros.

Este estudio, como se mencionó anteriormente en el objetivo, hace referencia a los resultados de las variables personales, ambientales, y de interacción social. 


\section{Métodos y materiales}

El presente trabajo se desarrolló bajo un enfoque cuantitativo de corte correlacional. Según Hernández, Fernández y Baptista (2010), este tipo de estudios permite conocer la relación o grado de asociación existente entre dos o más categorías o variables en un contexto específico. En este caso, se buscó conocer la relación de los factores personales, ambientales, y de interacción social con la intención de desertar o abandonar el programa de Bachillerato a Distancia - Virtual.

\subsection{Hipótesis}

Las hipótesis que se plantearon para este estudio fueron:

$\mathrm{H}_{1}$ : Los factores personales inciden significativamente en la intención de desertar del Bachillerato a Distancia - Virtual.

$\mathrm{H}_{2}$ : Los factores ambientales inciden significativamente en la intención de desertar del Bachillerato a Distancia - Virtual.

$\mathrm{H}_{3}$ : Los factores de interacción social inciden significativamente en la intención de desertar del Bachillerato a Distancia - Virtual.

\subsection{Unidad de análisis}

El contexto de estudio correspondió al programa de Bachillerato a Distancia - Virtual, ofertado por el Ministerio de Educación, y que es ejecutado a través de la Unidad Educativa Juan Montalvo de la ciudad de Quito. Estos colaboradores fueron las y los estudiantes matriculados legalmente en seis paralelos de primer año, y seis paralelos de tercer año, ambos del subnivel de Bachillerato General Unificado, y que al momento del estudio se encontraban cursando la cuarta y última unidad didáctica del periodo académico 2019 2020. La selección de los paralelos se basó en la accesibilidad del investigador, como docente de esta institución.

Se envió la invitación a participar de forma libre y voluntaria en el estudio a un total de 672 estudiantes, de los cuales respondieron 247 , lo que corresponde a un $36 \%$ de respuesta. Este porcentaje puede explicarse, en el sentido de que varios estudiantes, pese haber sido aceptados en el programa nunca han ingresado a la plataforma, y por otra parte, un número considerable de estudiantes han desertado en el transcurso de las tres unidades didácticas anteriores. Con la población de los 672 estudiantes, el tamaño de la muestra representativa es igual a 244 , no obstante, por conveniencia del investigador se trabajó con el total de los estudiantes que contestaron.

\subsection{Cuestionario - fuente de datos}

En este estudio se utilizó el cuestionario elaborado por Rivera (2011) en su tesis doctoral, quien desarrolló este instrumento para investigar las causas que influyen en la deserción en una Universidad a Distancia en Puerto Rico. De acuerdo con la autora, el instrumento ha sido validado, revisado y aprobado por un panel de expertos en investigación en educación, y por la junta de revisión institucional de la universidad investigada. Así también, la autora menciona que el instrumento obtuvo un valor de 0,932 para el alfa de Cronbach, reflejando un alto grado de confiabilidad (Rivera, 2011, p. 41).

El cuestionario en referencia se adecuó cuidadosamente a las características del Bachillerato a Distancia - Virtual. Con esta ligera adaptación el cuestionario constó de tres partes. Una primera que recogió aspectos demográficos (variables personales) mediante preguntas cerradas. La segunda parte constó de afirmaciones concernientes a las variables 
ambientales y de interacción social que debían ser respondidas con el grado de acuerdo o desacuerdo en escala Likert. La tercera parte presentó la pregunta de control que indagó en los estudiantes si han tenido o no la intención de desertar o abandonar los estudios. El cuestionario estuvo disponible durante 30 días en un sitio web, y la invitación se envió vía mensaje por la plataforma educativa Moodle a los participantes.

A continuación, en el Cuadro 1 se muestran las preguntas del cuestionario relacionadas con las variables personales, ambientales, y de interacción social. Así como también, la pregunta de control relacionada a la intención de abandonar o desertar del Bachillerato a Distancia Virtual. El número en el paréntesis hace referencia al número de pregunta original en el cuestionario de Rivera (2011).

\section{Variables personales}

\begin{tabular}{ll}
\hline $1(1)$ & Sexo \\
$2(5)$ & Zona de residencia \\
$3(2)$ & Edad (años) \\
$4(3)$ & Estado civil \\
5 & Número de hijos \\
$6(4)$ & Situación laboral \\
$7(6)$ & Ingreso individual mensual (dólares) \\
8 & Condición estudiantil \\
9 & Tiempo que ha dejado de estudiar (años) \\
$10(8)$ & Aspiración educativa \\
\hline
\end{tabular}

Variables ambientales

11 (32) Las responsabilidades familiares me imposibilitan para estudiar adecuadamente

12 (33) Cuento con suficientes recursos económicos para terminar el Bachillerato a Distancia - Virtual

13 (34) Mi situación económica representa una presión que obstaculiza mis estudios

14 (35) Mi familia me apoya en mis estudios

15 (36) Siento que he alcanzado un equilibrio entre mi hogar, mi empleo y mis estudios

\section{Variables de interacción social}

16 (38) En el Bachillerato a Distancia - Virtual me siento solo

17 (39) Los profesores del Bachillerato a Distancia - Virtual me transmiten entusiasmo para continuar en el curso 


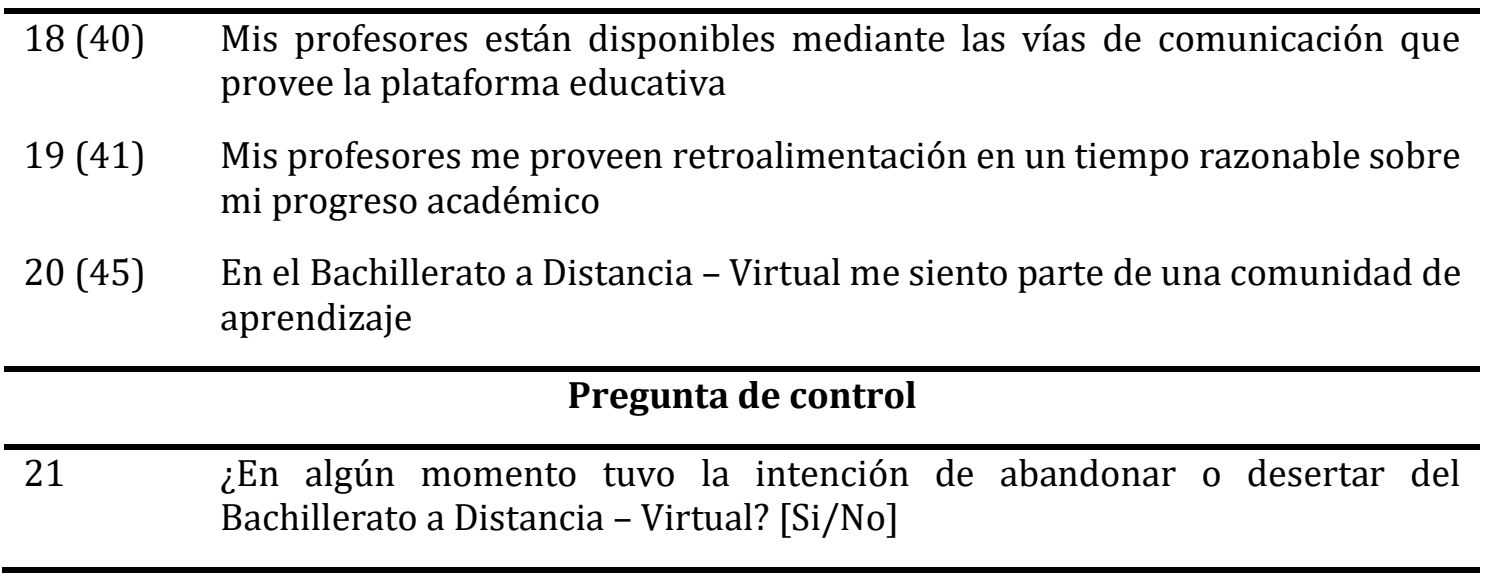

Cuadro 1. Preguntas del cuestionario

\subsection{Análisis de la información}

En un primer momento, con el fin de conocer las características demográficas de los participantes, el análisis de la información concerniente a las variables personales se realizó de forma descriptiva, esto permitió tener un acercamiento al perfil de la muestra. Además, se analizaron los resultados de la pregunta de control. En un segundo momento, y con el propósito de someter a prueba las hipótesis, se utilizó el programa estadístico IBM SPSS versión 25, en el cual se examinó, mediante tablas de contingencia (prueba Chi-Cuadrado), la asociación o independencia entre la pregunta de control y cada uno de los reactivos referentes a las variables personales, ambientales y de interacción social.

\section{Resultados}

\subsection{Descripción de la muestra}

En función de los resultados de las variables personales obtenidos con la aplicación del cuestionario, se determinó que el $51,5 \%$ de la muestra son mujeres, y el $48,5 \%$ son hombres. Además, el 76,7\% mencionó residir en la zona urbana, mientras que el 23,3\% en la zona rural. A su vez, el 51,4\% manifestó tener más de 30 años de edad. Por otro lado, el 64,1\% de los participantes aseveraron estar casados, y el 55,3\% afirmó tener más de un hijo. Con respecto a la situación laboral, el $48,5 \%$ de los participantes indicó tener un empleo a tiempo completo, sin embargo, el 51,5\% aseguró estar desempleado, o en su defecto laborar de manera informal. Así también, el 72,8\% declaró tener ingresos mensuales inferiores a USD 400. En relación al tiempo que han dejado de estudiar, el 57,2\% indicó tener menos de 10 años de rezago escolar, y con respecto a la aspiración educativa, el 85,4\% de los participantes manifestó deseos de continuar sus estudios a nivel superior.

\subsection{Pregunta de control}

A continuación, la Figura 1 muestra los resultados correspondientes a la pregunta de control referente a la intención de abandonar o desertar del Bachillerato a Distancia - Virtual. 


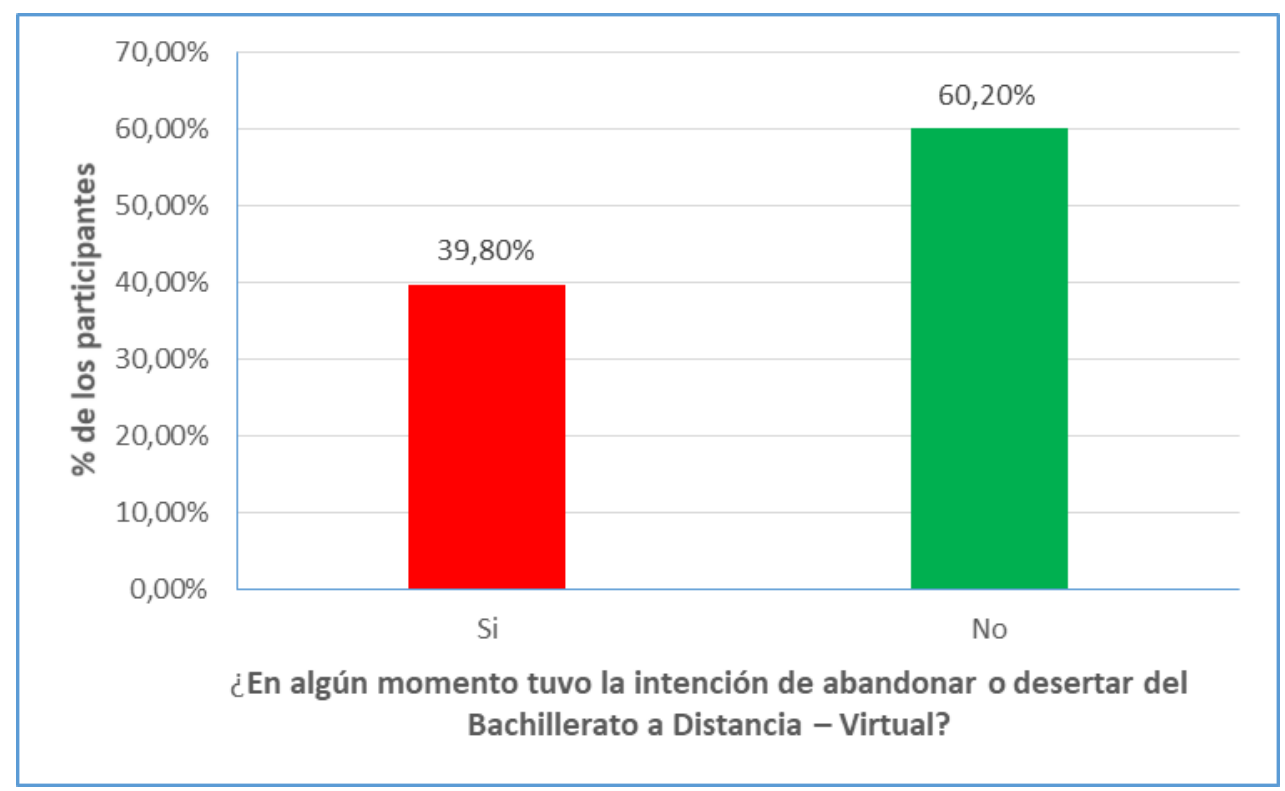

Figura 1. Intención de desertar del Bachillerato a Distancia - Virtual

Al preguntar a los participantes si en algún momento tuvieron la intención de abandonar o desertar del Bachillerato a Distancia - Virtual, un 60,2\% declaró no haber tenido la intención, sin embargo, el 39,8\% restante de los participantes manifestó si haber tenido la intención de desertar o abandonar los estudios. Este resultado es un signo de alerta para la institución, en el sentido de que 4 de cada 10 estudiantes tienen intenciones de abandonar el programa educativo.

\subsection{Prueba correlacional Chi-Cuadrado}

A continuación, se presentan los resultados de la prueba de correlación entre la intención de desertar del Bachillerato a Distancia - Virtual y las variables personales, ambientales y de interacción social, a partir de la prueba correlacional Chi-Cuadrado. Se determinó que de las diez variables personales, solo tres se correlacionaron de manera significativa (valor de significancia bilateral obtenido menor a 0,05 ) con la intención de desertar o abandonar los estudios. De igual forma, se comprobó que apenas una de las cinco variables ambientales se correlacionó de manera significativa con la intención de desertar. Por otra parte, se encontró que cuatro de las cinco variables de interacción social se correlacionaron significativamente con la intención de desertar o abandonar el Bachillerato a Distancia Virtual. En el Cuadro 2 se muestra el resumen de los resultados. 


\begin{tabular}{|c|c|c|c|}
\hline \multicolumn{4}{|c|}{ Chi-Cuadrado de Pearson } \\
\hline $\begin{array}{l}\text { Variables personales, ambientales y de } \\
\text { interacción social. }\end{array}$ & Valor & $\begin{array}{r}\text { Signif } \\
\text { asin } \\
\text { (bila }\end{array}$ & $\begin{array}{l}\text { ción } \\
\text { ca } \\
\text { al) }\end{array}$ \\
\hline Sexo & 5,652 & 0,217 & \\
\hline Zona de residencia & 6,726 & 0,010 & $* *$ \\
\hline Edad & 2,471 & 0,116 & \\
\hline Estado civil & 11,019 & 0,001 & $* *$ \\
\hline № de hijos & 14,212 & 0,000 & $* *$ \\
\hline Ingreso individual mensual & 3,518 & 0,061 & \\
\hline Condición estudiantil & 0,968 & 0,325 & \\
\hline Años de rezago escolar & 3,332 & 0,068 & \\
\hline Aspiración educativa & 0,345 & 0,557 & \\
\hline Situación laboral & 0,002 & 0,969 & \\
\hline $\begin{array}{l}\text { Las responsabilidades familiares me imposibilitan } \\
\text { para estudiar adecuadamente }\end{array}$ & 0,022 & 0,881 & \\
\hline $\begin{array}{l}\text { Cuento con suficientes recursos económicos para } \\
\text { terminar mi Bachillerato a Distancia - Virtual }\end{array}$ & 10,952 & 0,001 & $* *$ \\
\hline $\begin{array}{l}\text { Mi situación económica representa una presión } \\
\text { que obstaculiza mis estudios }\end{array}$ & 0,873 & 0,350 & \\
\hline Mi familia me apoya en mis estudios & 4,052 & 0,064 & \\
\hline $\begin{array}{l}\text { Siento que he alcanzado un equilibrio entre mi } \\
\text { hogar, mi empleo y mis estudios }\end{array}$ & 1,076 & 0,299 & \\
\hline $\begin{array}{l}\text { En el Bachillerato a Distancia - Virtual me siento } \\
\text { solo }\end{array}$ & 6,293 & 0,012 & $* *$ \\
\hline $\begin{array}{l}\text { Los profesores del Bachillerato a Distancia - } \\
\text { Virtual me transmiten entusiasmo para continuar } \\
\text { en el curso }\end{array}$ & 0,468 & 0,014 & $* *$ \\
\hline $\begin{array}{l}\text { Mis profesores están disponibles mediante las vías } \\
\text { de comunicación que provee la plataforma } \\
\text { educativa }\end{array}$ & 5,038 & 0,025 & $* *$ \\
\hline $\begin{array}{l}\text { Mis profesores me proveen retroalimentación en } \\
\text { un tiempo razonable sobre mi progreso académico }\end{array}$ & 6,293 & 0,012 & $* *$ \\
\hline $\begin{array}{l}\text { En el Bachillerato a Distancia - Virtual me siento } \\
\text { parte de una comunidad de aprendizaje }\end{array}$ & 0,942 & 0,332 & \\
\hline
\end{tabular}


Adicionalmente, con la finalidad de interpretar estos resultados, se utilizó un modelo de regresión logística binaria, para ello fue necesario recodificar las variables personales, ambientales y de interacción social (independientes) a variables dicotómicas o binarias, 1 y 0 . Asimismo, en el modelo, la variable dependiente es binaria y toma el valor de 1 si el estudiante ha tenido la intención de desertar o abandonar el Bachillerato a Distancia Virtual. De acuerdo con los resultados se encontró que:

- Existe una relación estadísticamente significativa entre la zona de residencia y la intención de desertar. Conforme a la recodificación, en donde la variable toma el valor de 1 si el estudiante reside en la zona urbana, se interpreta que las y los estudiantes que residen en la zona urbana tienen menor probabilidad de desertar 0 abandonar el Bachillerato a Distancia - Virtual que aquellos que viven en zonas rurales.

- Entre el estado civil de los estudiantes y la intención de desertar del Bachillerato a Distancia - Virtual existe una relación estadísticamente significativa. De acuerdo con la recodificación, la variable toma el valor de 1 si el estudiante es casado, por tanto, se interpreta que las y los estudiantes que están casados son más propensos a desertar o abandonar los estudios que aquellos que no lo están.

- Existe una relación significativa entre el número de hijos y la intención de desertar o abandonar los estudios. Acorde con la recodificación, en donde la variable toma el valor de 1 si el estudiante no tiene hijos o tiene máximo uno, se interpreta que las y los estudiantes que no tienen hijos, o tienen máximo uno, tienen menor probabilidad de abandonar o desertar de los estudios que aquellos estudiantes que tienen más de un hijo.

- Entre la intención de desertar del Bachillerato a Distancia - Virtual y contar con suficientes recursos económicos para terminar los estudios existe una relación estadísticamente significativa. Conforme a la recodificación, en donde la variable toma el valor de 1 si el estudiante está de acuerdo con la aseveración, se interpreta que las y los estudiantes que cuentan con los recursos económicos suficientes son menos probables a desertar de los estudios que aquellos que no los tienen.

- Existe una relación estadísticamente significativa entre la intención de desertar y sentirse solo en el Bachillerato a Distancia - Virtual. Conforme a la recodificación, en donde la variable toma el valor de 1 si el estudiante está de acuerdo con la aseveración, se interpreta que las y los estudiantes que se sienten solos o abandonados son más probables a desertar de los estudios que aquellos que no sienten soledad.

- Entre la intención de desertar y el entusiasmo que transmiten los docentes del Bachillerato a Distancia - Virtual existe una relación estadísticamente significativa. Conforme a la recodificación, en donde la variable toma el valor de 1 si el estudiante está de acuerdo con la aseveración, se interpreta que las y los estudiantes cuyos profesores les transmiten entusiasmo son menos probables a desertar de los estudios que aquellos cuyos docentes no les comunican entusiasmo o motivación.

- Existe una relación estadísticamente significativa entre la disponibilidad de los docentes a través de la plataforma educativa y la intención de desertar. Conforme a la recodificación, en donde la variable toma el valor de 1 si el estudiante está de acuerdo con la aseveración, se interpreta que las y los estudiantes cuyos profesores están disponibles a través de la plataforma educativa son menos probables a desertar de los estudios que aquellos estudiantes cuyos docentes no lo están. 
- Entre la intención de desertar del Bachillerato a Distancia - Virtual y la retroalimentación oportuna que proveen los docentes existe una relación estadísticamente significativa. Conforme a la recodificación, en donde la variable toma el valor de 1 si el estudiante está de acuerdo con la aseveración, se interpreta que las y los estudiantes que reciben retroalimentación oportuna de parte de los docentes son menos probables a desertar de los estudios que aquellos que no la reciben.

En contraste con todo lo anterior, los resultados estadísticos de las otras siete variables personales: sexo, edad, ingresos, condición estudiantil, rezago escolar, aspiración educativa, y situación laboral, mostraron que los valores de significancia bilateral superan el 0,05; por lo tanto, se deduce que no existe relación estadísticamente significativa entre dichos factores personales y la intención de desertar del Bachillerato a Distancia - Virtual. De igual forma, los resultados de las otras cuatro variables ambientales: responsabilidades familiares, situación económica, apoyo familiar, y equilibrio entre empleo-hogar-estudios, expresaron que los valores de significancia bilateral también son mayores a 0,05 ; en consecuencia, tampoco existe relación estadísticamente significativa entre estos factores y la intención de desertar. En cambio, por el lado de los factores de interacción social, sentirse parte de una comunidad de aprendizaje fue la única variable cuyo valor de significancia bilateral superó a 0,05 ; por tanto, no se relaciona significativamente con la intención de desertar o abandonar los estudios.

\section{Discusión}

\subsection{Factores personales}

De acuerdo con los resultados de esta investigación, los estudiantes del Bachillerato a Distancia - Virtual que residen en zonas urbanas tienen menor probabilidad de desertar que aquellos que residen en zonas rurales. Este resultado se confirma con los hallazgos del Instituto Nacional de Evaluación Educativa (2018), quien mencionó que en el año 2017 la tasa de deserción en Bachillerato fue menor en la zona urbana (4,3\%) que en la zona rural $(5,5 \%)$. Este resultado podría estar relacionado al hecho de que todavía existen zonas rurales en las cuales se dificulta el acceso a internet, recurso indispensable para estudiar en el Bachillerato a Distancia - Virtual.

Asimismo, con respecto al estado civil, los resultados demuestran que los estudiantes que están casados tienen mayor probabilidad de abandonar o desertar del Bachillerato a Distancia - Virtual. Este hallazgo ratifica los resultados encontrados por Ruiz, García y Pérez (2014), quienes determinaron en su estudio desarrollado en el Bachillerato Virtual de la Universidad Autónoma de Sinaloa, que uno de los factores personales que más influye en la deserción escolar es estar casado. Esto probablemente se debe a que los estudiantes casados dedican mayor tiempo a las responsabilidades familiares, y no necesariamente a las actividades escolares.

La última de las variables personales que demuestra relación con la deserción escolar del Bachillerato a Distancia - Virtual, es el número de hijos. Esta condición, casi de forma similar a la anterior, podría entenderse en el sentido de que los estudiantes que tienen varios hijos están obligados a trabajar prácticamente a tiempo completo para poder responder a las necesidades de sus hijos, además del tiempo que deben dedicar al cuidado y crianza de los mismos. Esta particularidad disminuye el tiempo que pueden dedicar a los estudios con respecto a aquellos estudiantes que no tienen hijos. Esta condición aumenta la probabilidad de tomar la decisión de desertar.

Licencia Creative Commons Atribución 4.0 Internacional (CC BY 4.0)

Revista Cátedra, 3(3), pp. 111-128, septiembre-diciembre 2020. e-ISSN:2631-2875 https://doi.org/10.29166/catedra.v3i3.2279 


\subsection{Factores ambientales}

Por el lado de los factores ambientales, la única variable que se relaciona de forma significativa con la intención de desertar es la referente a contar con suficientes recursos económicos para terminar el Bachillerato a Distancia - Virtual. Este resultado corrobora los hallazgos de Ruiz, García y Pérez (2014), quienes determinaron en su estudio que el factor económico está entre las primeras causas detonantes de la deserción escolar. Esto podría estar relacionado, principalmente, a la escasez de dinero, lo que repercute en falta de recursos para poder culminar los estudios.

\subsection{Factores de interacción social}

Con respecto a los factores de interacción social, los resultados muestran que el sentimiento de soledad incide significativamente en la intención de desertar o abandonar el Bachillerato a Distancia - Virtual. Este hallazgo confirma los resultados de Capera (2015), quien concluyó en su estudio sobre factores que influyen en la deserción escolar en educación a distancia, realizada en una Universidad de Colombia, que el sentimiento de soledad y abandono por parte de los docentes y la institución son los principales factores que influyen en la deserción escolar. Este resultado podría estar relacionado al escaso seguimiento y acompañamiento por parte de los docentes tutores y de la misma institución.

Igualmente, los resultados muestran que el entusiasmo y la motivación que transmiten los docentes a los estudiantes inciden significativamente en la intención de desertar del Bachillerato a Distancia - Virtual. Este hallazgo corrobora los resultados de Bryndum y Jerónimo (2005), quienes concluyeron en su estudio sobre la motivación en entornos telemáticos, que la motivación es un componente fuertemente importante en la modalidad a distancia virtual, ya que sin ella el resto de componentes se debilitan al punto de que se puede producir el abandono o deserción del estudiante.

Así también, los hallazgos demuestran que la retroalimentación oportuna que proveen los profesores incide significativamente en la intención de desertar del Bachillerato a Distancia - Virtual. Este resultado ratifica los resultados de García, Guajardo y Valdez, (2014), quienes encontraron en su estudio acerca de la retroalimentación como herramienta para la interacción, que la falta o escasa retroalimentación por parte de los docentes influye significativamente en la deserción escolar en la educación a distancia virtual.

Asimismo, los resultados indican que la disponibilidad de los profesores a través de la plataforma educativa influye significativamente en la decisión de desertar del Bachillerato a Distancia - Virtual. Este hallazgo confirma los resultados de González (2017), quien concluyó en su estudio acerca de factores que favorecen la presencia docente en entornos virtuales, que la permanencia o abandono de los estudiantes en los programas de educación virtual está en correspondencia con la mayor o menor disponibilidad y presencia de los docentes a lo largo del proceso educativo.

\section{Conclusiones}

Los resultados de la presente investigación sobre la incidencia de los factores personales, ambientales, y de interacción social en la deserción escolar del Bachillerato a Distancia Virtual de la Unidad Educativa Juan Montalvo, permitieron plantear las siguientes conclusiones:

Los factores de interacción social son los de mayor incidencia en la intención de desertar o abandonar el Bachillerato a Distancia - Virtual. Entre estos se destacan el sentimiento de soledad, el poco entusiasmo y motivación que transmiten los docentes, la falta de una 
retroalimentación oportuna, y la escasa disponibilidad de los profesores a través de la plataforma educativa. Este resultado ratifica los hallazgos de Rivera (2011), quien concluyó en su estudio que los factores más influyentes de la deserción son los referentes a la interacción social y académica. En conclusión, se acepta la $\mathrm{H}_{3}$ que expresa: los factores de interacción social inciden significativamente en la intención de desertar del Bachillerato a Distancia - Virtual.

Por el lado de los factores personales, residir en la zona rural, estar casado, y tener más de un hijo son los únicos factores que inciden significativamente en la intención de desertar. No obstante, se determinó que las otras siete variables personales bajo estudio no inciden significativamente en la intención de desertar o abandonar los estudios. Este resultado corrobora los hallazgos de Capera (2015), quien encontró que las variables personales no inciden mayormente en la deserción escolar. Por lo tanto, se rechaza la $\mathrm{H}_{1}$ y se concluye que los factores personales no inciden significativamente en la intención de desertar del Bachillerato a Distancia - Virtual.

De forma similar, los resultados muestran que contar con suficientes recursos económicos es el único de los factores ambientales que incide significativamente en la intención de desertar. Sin embargo, se demostró que los otros cuatro factores ambientales bajo estudio no inciden significativamente en la deserción escolar. Por lo tanto, también se rechaza la $\mathrm{H}_{2}$, y se concluye que los factores ambientales no inciden significativamente en la intención de desertar del Bachillerato a Distancia - Virtual.

En definitiva, en la presente investigación se encontró que los factores de interacción social si inciden significativamente en la intención de desertar o abandonar el Bachillerato a Distancia - Virtual. En contraste, se determinó que los factores personales y ambientales no inciden mayormente en la deserción. Estos resultados son un síntoma de alarma para la institución, debido a que, de acuerdo con esta investigación, los estudiantes previenen y solventan las problemáticas relacionadas a su entorno personal y ambiental y evitan que tales factores dificulten su permanencia en el programa educativo. Esto permite deducir que las causas de la deserción escolar responden a otros factores.

Finalmente, considerando que este trabajo se desarrolló bajo el marco teórico de Rivera (2011), y que en la presente investigación se estudiaron únicamente los factores personales, ambientales y de interacción social, es necesario que futuras investigaciones analicen las otras categorías de variables planteadas por la autora, es decir, se investigue la relación entre la deserción escolar y los factores académicos y de apoyo tecnológico. Así también, acercamientos cualitativos a las realidades de los estudiantes, docentes y autoridades, podrían proveer testimonios que permitan identificar otros factores que inciden en la deserción escolar y que afectan a este programa educativo.

Licencia Creative Commons Atribución 4.0 Internacional (CC BY 4.0)

Revista Cátedra, 3(3), pp. 111-128, septiembre-diciembre 2020. e-ISSN:2631-2875

https://doi.org/10.29166/catedra.v3i3.2279 


\section{Bibliografía}

Acuña Escobar, C. E. (2018). Deserción escolar en educación a distancia: datos de estudio. Revista Vinculando, 1-10. Recuperado de https://www.researchgate.net/publication/330599406 LA DESERCION ESCOLA R EN LA EDUCACION A DISTANCIA

Antamba, L. (2015). Desagregación socio-demográfica de la tasa de abandono a primer año del bachillerato. Contexto, Análisis de indicadores educativos, 1, 15-29. Recuperado de

https://educacion.gob.ec/wpcontent/uploads/downloads/2016/01/Publicaciones/PUB ContextoVol1 may201 $\underline{5 . p d f}$

Benítez Zavala, A. (2016). Abandono escolar: modelos que lo explican y programas para atenderlo. Una revisión crítica. Revista de Educación y Desarrollo, 37, 27-32. Recuperado http://www.cucs.udg.mx/revistas/edu desarrollo/anteriores/37/37 Benitez.pdf

Bryndum, S., \& Jerónimo, J. (2005). La motivación en los entornos telemáticos. RED. Revista de Educación a Distancia, V(13), 1-24. Recuperado de https://revistas.um.es/red/article/view/24381/23721

Capera Urrego, A. I. (2015). Influencia de los factores personales y académicos sobre la deserción de estudiantes en la educación a distancia. Revista de Investigaciones UNAD, 14(1), 61. Recuperado de https://www.researchgate.net/publication/318353542 Influencia de los factores personales y academicos sobre la desercion de estudiantes en la educacion a distancia

Cruz Garza, M. E. (2003). Deserción escolar, factores dependientes de la institución educativa, en estudiantes de la carrera de Químico Farmacéutico Biologo de la Facultad de Ciencias Químicas de la Universidad Autónoma de Nueva León (Universidad Autónoma de Nueva León). Recuperado de http://eprints.uanl.mx/5350/1/1020149157.PDF

De Aguinaga Vázquez, C. P., \& Barragán de Anda, A. B. (2007). La presencia social como elemento minimizador de la deserción en la educación a distancia. Virtual Educa Brasil. Recuperado https://reposital.cuaed.unam.mx:8443/xmlui/bitstream/handle/20.500.12579/1 455/38-CDV.PDF? sequence $=1$

Espíndola, E., \& León, A. (2002). La deserción escolar en América Latina: un tema prioritario para la agenda regional. Revista Iberoamericana de Educación, 30, 39-62. Recuperado de http://www.seg.guanajuato.gob.mx/Ceducativa/CartillaB/6antologia/antecedente s/pdf/32.- LA DESERCIÓN ESCOLAR EN AMÉRICA LATINA UN TEMA PRIORITARIO PARA LA AGENDA REGIONAL.pdf

Frankola, K. (2001). Why Online Learners Drop Out. Workforce Management, 80(2), 53-58. Recuperado de https://coursecontent.ntc.edu/synchronous/why-online-learnersdrop-ou.pdf

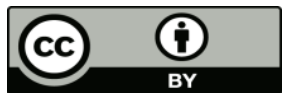


García Aretio, L. (2019). El problema del abandono en estudios a distancia. Respuestas desde el Diálogo Didáctico Mediado. RIED. Revista Iberoamericana de Educación a Distancia, 22(1), 245. Recuperado de http://revistas.uned.es/index.php/ried/article/view/22433

García, D., Guajardo, V., \& Valdez, F. (2014). La Retroalimentación como factor indispensable en la educación on-line. VI Congreso Internacional Latina de Comunicación Social VI CILCS - Universidad de La Laguna, 1-21. Recuperado de http://www.revistalatinacs.org/14SLCS/2014 actas/071 Garcia.pdf

González, G. de P. (2017). Factores que favorecen la presencia docente en entornos virtuales de aprendizaje. Tendencias pedagógicas, (29), 43-58. Recuperado de https://repositorio.uam.es/handle/10486/676818

González Lira, A. M. (2002). Reflexiones sobre los factores que influyen en la desercion escolar del adolescente (Universidad Pedaógica Nacional). Recuperado de http://200.23.113.51/pdf/19411.pdf

Hernández Sampieri, R., Fernández Collado, C., \& Baptista Lucio, M. (2010). Metodología de la investigación (Quinta; S. A. D. C. V. McGRAW-HILL / INTERAMERICANA EDITORES, Ed.). Recuperado de https://www.esup.edu.pe/descargas/dep investigacion/Metodologia de la investigación 5ta Edición.pdf

Instituto Nacional de Evaluación Educativa. (2018). La educación en Ecuador: Logros alcanzados y nuevos desafíos Resultados educativos 2017-2018. En Ministerio de Educación. Recuperado de www.evaluacion.gob.ec

Morfin Otero, M., González Quintanilla, C. A., \& Palomera Pérez, B. J. (2018). Deserción en un programa en línea de cuatro ciclos. Revista EDUCATECONCIENCIA, 18(1), 39-64. Recuperado de http://tecnocientifica.com.mx/educateconciencia/index.php/revistaeducate/articl e/view/463/521

Muñoz Barreneche, C. F. (2013). Deserción escolar, un concepto que no concluye: Casos de no conclusión satisfactoria del ciclo escolar en la Institución Educativa De Santa Librada (Universidad del Valle). Recuperado de http://bibliotecadigital.univalle.edu.co/bitstream/10893/6446/1/0461928-p.pdf

Peña Quintal, E. C., \& Rodríguez Góngora, F. C. (2019). Estrategias para disminuir la deserción escolar en el Bachillerato en Línea de la Universidad Autónoma de Yucatán. Revista Mexicana de Bachillerato a Distancia, (21), 71-80. Recuperado de http://revistas.unam.mx/index.php/rmbd/article/view/68533

Peralta Castro, R., \& Mora Rodríguez, J. (2016). El abandono en la educación virtual y a distancia: el caso de la Universidad Nacional Abierta y a Distancia-UNAD, Colombia. VI CLABES Sexta Conferencia Latinoamericana sobre el Abandono en la Educación Superior, 1-10. Recuperado de https://revistas.utp.ac.pa/index.php/clabes/article/view/1330/1819

Posligua Anchundia, R. (2019). La realidad educativa y social ecuatoriana a partir del análisis de la deserción académica. ReHuSo: Revista de Ciencias Humanísticas y Sociales, 36-45. Recuperado de https://dialnet.unirioja.es/servlet/articulo?codigo=7047159 
Pugo Pugo, S. X. (2017). Causas que incidieron en la deserción estudiantil en la básica superior del Colegio Particular a Distancia Mario Rizzini de la ciudad de Cuenca, periodo 2015-2016 (Universidad Politécnica Salesiana, Sede Cuenca). Recuperado de https://dspace.ups.edu.ec/bitstream/123456789/14500/5/UPS-CT007142.pdf

Rivera Montalvo, D. E. (2011). Factores que Inciden en la Retención o Deserción del Estudiante a Distancia (Nova Southeastern University). Recuperado de http://ponce.inter.edu/cai/tesis/derivera/index.pdf

Ruiz Ramírez, R., García Cué, J. L., \& Pérez Olvera, M. A. (2014). Causas y consecuencias de la deserción escolar en el bachillerato: caso Universidad Autónoma de Sinaloa. Ra Ximhai, 51-74. Recuperado de https://www.redalyc.org/pdf/461/46132134004.pdf

Tinto, V. (1982). Limits of Theory and Practice in Student Attrition. The Journal of Higher Education, 53(6), 687. Recuperado de http://www.sigmus.edu.rs/eng/files/Limits of Theory and Practice in Student Attrition.pdf

Tinto, V. (1989). Definir la deserción: una cuestión de perspectiva. Revista de Educación Superior, ANUIES, MEXICO, 71, 1-9. Recuperado de http://publicaciones.anuies.mx/pdfs/revista/Revista71 S1A3ES.pdf

Vásquez Martínez, C. R., \& Rodríguez Pérez, M. C. (2007). La deserción estudiantil en educación superior a distancia: perspectiva teórica y factores de incidencia. Revista latinoamericana de estudios educativos, 37(3), 107-122. Recuperado de https://www.redalyc.org/articulo.oa?id=27011410005

Venegas Alvarez, G., Chiluisa Chiluisa, M., Castro Bungacho, S., y Casillas, I. (2017). La deserción en la educación. Revista Boletín Redipe, 6(4), 235-239. Recuperado de https://revista.redipe.org/index.php/1/article/view/240 


\section{Autores}

ANGEL ANDRÉS VIVANCO-SARAGURO obtuvo en 2017 su título de Licenciado en Ciencias de la Educación mención Matemática y Física por la Facultad de Filosofía, Letras y Ciencias de la Educación de la Universidad Central del Ecuador. En 2019 obtuvo su título de Especialista Superior en Educación y Nuevas Tecnologías de la Información y Comunicación por la Universidad Andina Simón Bolívar, Sede Ecuador. El suscribiente está en la fase de elaboración del trabajo final del programa de Maestría de Investigación en Educación en la Universidad Andina Simón Bolívar, Sede Ecuador.

En los últimos años se ha desempeñado como docente de enseñanza media. Ha trabajado como compositor de ítems para los proyectos del Instituto Nacional de Evaluación Educativa, en las áreas de matemática y razonamiento numérico y abstracto. Actualmente es docente en el Bachillerato a Distancia - Virtual de la Unidad Educativa Juan Montalvo de la Ciudad de Quito, donde imparte las asignaturas de Matemática y Física. 